\title{
Freestanding waveguides in silicon
}

\author{
P. Y. Yang, ${ }^{\text {a) }}$ G. Z. Mashanovich, I. Gomez-Morilla, W. R. Headley, and G. T. Reed \\ Advanced Technology Institute, School of Electronics and Physical Sciences, University of Surrey, Guildford, \\ Surrey GU2 7XH, United Kingdom
}

\author{
E. J. Teo and D. J. Blackwood \\ Department of Materials Science and Engineering, 7 Engineering Drive 1, National University of \\ Singapore, Singapore 117574, Singapore
}

M. B. H. Breese and A. A. Bettiol

Department of Physics, 2 Science Drive 3, National University of Singapore, Singapore 117542, Singapore

(Received 12 January 2007; accepted 22 May 2007; published online 13 June 2007)

\begin{abstract}
Using a direct-write process for the production of three dimensional microstructures on a semiconductor, freestanding waveguides have been realized in silicon. The waveguides are produced by a focused beam of high energy protons that is scanned over a silicon substrate. The latent image of the scan is subsequently developed by electrochemical etching. Herein the authors report on the fabrication method as well as determining the propagation loss of these structures. Propagation loss values of 13.4 and $14.6 \mathrm{~dB} / \mathrm{cm}$ were obtained for these preliminary structures for transverse electric and transverse magnetic polarizations, respectively. (C) 2007 American Institute of Physics. [DOI: 10.1063/1.2749175]
\end{abstract}

Silicon photonics is experiencing a dramatic increase in interest due to emerging application areas and several breakthrough in device and technology development. ${ }^{1-5}$ Most conventional waveguides in silicon photonics are fabricated in the silicon-on-insulator (SOI) material system in the form of a strip or a rib waveguide. These waveguides, however, are not suitable for longer wavelengths (except in the 3-3.5 $\mu \mathrm{m}$ range) due to the absorption of the silicon dioxide insulating layer. ${ }^{6}$ Silicon-based long wave infrared photonics could find applications in several areas including sensing, communications, signal processing, missile detection, and imaging. To address these wavelengths, several different waveguide structures have been investigated such as photonic crystal, plasmon optic, hollow, and nanoslotted rib or strip waveguides. In this letter, the fabrication and propagation loss measurements of a different structure, the freestanding waveguide, are reported. As this waveguide has an air cladding, it is a viable structure for long wavelength applications. ${ }^{6}$ Alternatively, any other suitable cladding could easily be deposited to make the structures suitable for specific wavelength ranges.

Proton beam writing $(\mathrm{PBW})$ is an advanced lithographic technique for micromachining on the submicron scale. PBW utilizes a high energy ion microbeam to irradiate suitable resists [e.g., SU-8 and polymethyl methcrylate (PMMA)]. Subsequent wet etching is then employed to develop the resist. While it was initially investigated for producing latent microstructures in high molecular weight PMMA resist, Polesello et al. ${ }^{7}$ and Mistry et al. ${ }^{8}$ have demonstrated PBW as a method to fabricate three dimensional structures directly in $\mathrm{Si}$ and GaAs, respectively, thereby eliminating the need for a resist.

There are several potential advantages of PBW. It is a direct-write process which eliminates the need for a potentially costly mask. Furthermore, prototype devices which require small structural modifications can be produced by sim-

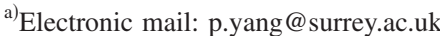

ply modifying the scan of the microbeam whereas modifications to a device using photolithographic methods would require the layout and fabrication of an additional mask.

A second benefit is that the protons are deposited in the semiconducting material in a well-defined range, thereby allowing for good vertical control of the fabrication of devices. Furthermore, the high energy protons deviate little from the ideal straight path due to multiple small-angle Coulomb scattering. The result is that much smaller lateral straggle occurs as compared to e-beam lithography and hence a tighter control on the lateral dimensions of the structure.

Proton beam writing has already demonstrated its potential to be a next generation lithography technique in siliconbased applications. Recently freestanding bridges, multilevel structures, and high aspect-ratio nanotips fabricated in silicon have been demonstrated by Breese et al. ${ }^{9}$ and Teo et al. ${ }^{10}$ Typically, a finely focused $\mathrm{MeV}$ proton beam is selectively scanned over the silicon surface. The high energy protons penetrate the silicon substrate and stop within a well-defined range. The depth is controlled by the energy of the protons. As the protons propagate through the silicon, they undergo a series of collisions with electrons. Protons are deflected away from an ideal straight path, resulting in a beam cross section increasing in size with depth. The energy deposited due to nuclear collisions per unit length is fairly constant during the first half of the penetration depth but increases sharply at the end of range, because the speed of protons is much lower than their initial value, thereby increasing the probability of nuclear collisions with target atoms. The penetration range and energy deposition profiles can be simulated using SUSPRE, ${ }^{11}$ an ion implantation simulation package. High energy protons lose their energy and eventually come to rest below the surface. Silicon vacancies are created along the path of the protons, with most of the damage occurring towards the end of their range. After irradiation, the sample is electrochemically etched in a solution of hydrofluoric acid $(\mathrm{HF})$ :water:ethanol in a ratio of $1: 1: 2$. Once the silicon be- 


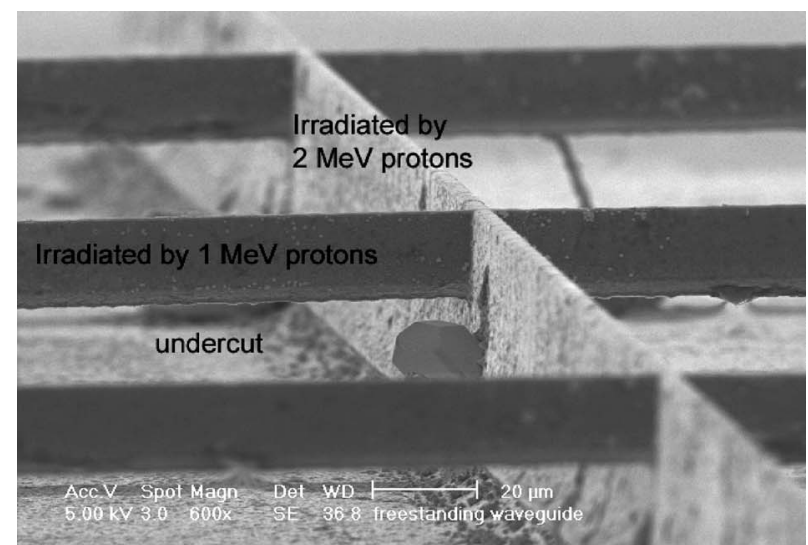

(a)

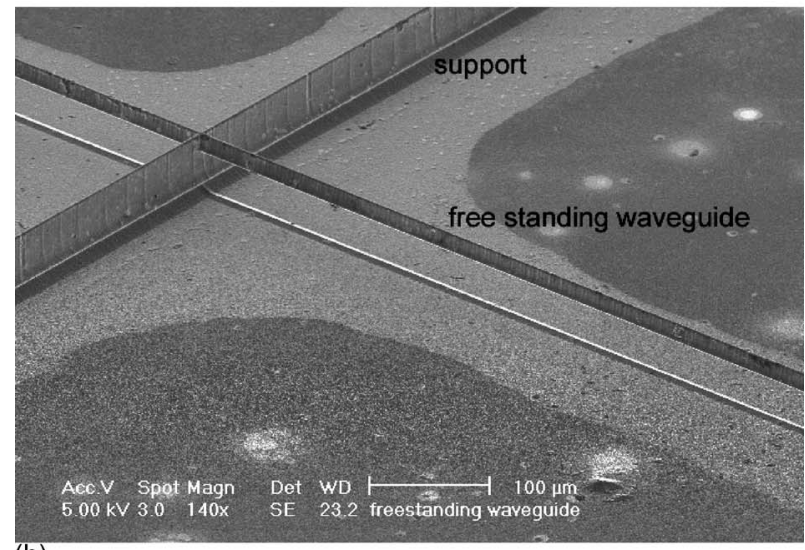

(b)

FIG. 1. (a) Sideview of $1 \mathrm{MeV}$ implanted waveguide in relation to a $2 \mathrm{MeV}$ pillar demonstrating the undercutting of the waveguide. (b) Freestanding waveguide suspended from the silicon substrate by the pillars.

comes porous, it can be removed by a dilute potassium hydroxide solution. In the irradiated regions, it is important that the density of defects at the surface of the silicon be high enough to significantly reduce the electrical hole current flow and therefore inhibit the electrochemical reaction at the damaged silicon-electrolyte interface. The result is a significant reduction in the rate of producing porous silicon in the proton irradiation regions as compared to the unirradiated region. The final structure produced after etching is a three dimensional representation of the scanned area. This area determines the dimensions of the final structure. The height of the structure is controlled by the etching time and current.

In order to fabricate freestanding waveguides, two different ion energies are required. A high energy implant is used to create pillars upon which the waveguides will be supported. A lower energy implant is subsequently used to create the waveguides. Based the simulation results obtained by SUSPRE, $2 \mathrm{MeV}$ protons travel approximately $50 \mu \mathrm{m}$ into silicon and $1 \mathrm{MeV}$ protons penetrate approximately $17 \mu \mathrm{m}$. The pillars were therefore created by irradiating the silicon with a dose of $0.7 \times 10^{15}$ protons $/ \mathrm{cm}^{2}$ at an energy of $2 \mathrm{MeV}$ and the waveguides with a dose of $0.6 \times 10^{15}$ protons $/ \mathrm{cm}^{2}$ at an energy of $1 \mathrm{MeV}$. Two scans of the microbeam were made over the same position in order to create the localized damage pattern. The doses are significant enough to inhibit the current flow in the irradiated regions, thereby limiting the formation process of porous silicon during electrochemical

[Fig. 1(b)].
etching.
Downloaded 09 Dec 2010 to 131.227.3.190. Redistribution subject to AIP license or copyright; see http://apl.aip.org/about/rights_and_permissions

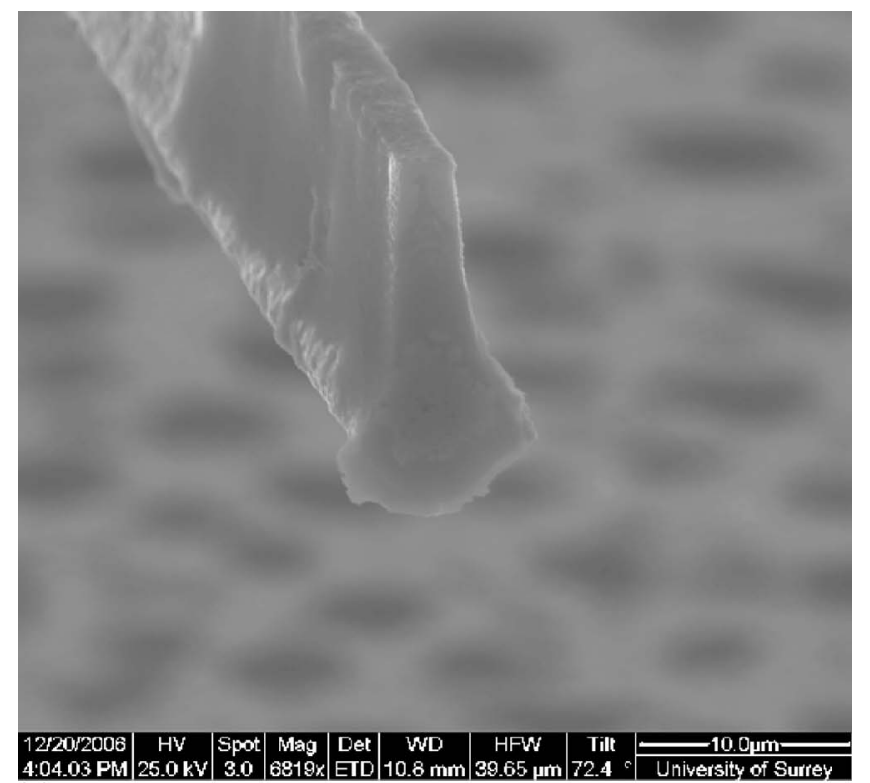

(a)

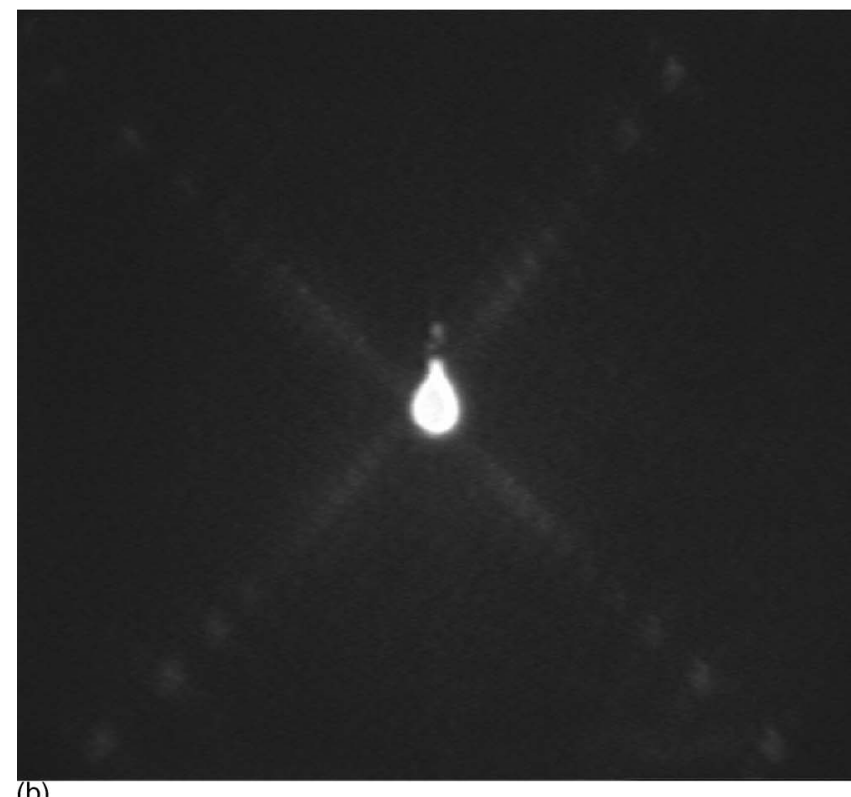

(b)

FIG. 2. (a) SEM of the freestanding waveguide facet. (b) Optical output from a freestanding waveguide imaged with an IR sensitive camera.

The electrochemical etch rate is mainly dependent on three important parameters: dopant type and concentration, HF concentration, and applied current density. In order to remove approximately $50 \mu \mathrm{m}$ of bulk unirradiated silicon, the sample was etched for 20 min thus giving a removal rate of approximately $2.5 \mu \mathrm{m} / \mathrm{min}$. Initially, the etching occurs in all regions except in the irradiated portions. As the etch continues, it will begin to undercut the unirradiated region that lies underneath the $1 \mathrm{MeV}$ irradiated region. The $2 \mathrm{MeV}$ regions will continue to remain unetched thereby creating the pillars to hold the waveguides. In this way, we can fabricate the freestanding waveguide structures in a single etch step. Figure 1(a) shows how the etching goes beyond the range of the $1 \mathrm{MeV}$ protons $(\sim 17 \mu \mathrm{m})$, where undercutting of the waveguides occurs. ${ }^{10}$ In the end, the waveguides are fully detached from the substrate and supported only by the pillars [Fig. 1(b)]. 
A scanning electron micrograph (SEM) of the waveguide cross section is shown in Fig. 2(a). The waveguide has a "tear-drop" shape with a height of $17 \mu \mathrm{m}$ and width of $3.7 \mu \mathrm{m}$ at the top and $8 \mu \mathrm{m}$ at the bottom. This shape is due to lateral spreading of the beam with depth, but it can be altered by alternative choices of irradiation energies.

Propagation loss measurements were performed at the wavelength of $1550 \mathrm{~nm}$ in order to compare it with the loss of standard (SOI) silicon waveguides prevalent in the literature. A free space $1550 \mathrm{~nm}$ laser was coupled to the freestanding waveguides using an objective lens. Prior to the objective lens a polarizing beam splitter and half-wave plate were inserted into the beam path which enabled discrimination between the TE and TM polarizations. The light output from the waveguides was collected by another objective lens and imaged using an IR sensitive camera [Fig. 2(b)]. The optical power transmitted by the waveguide was also measured with a power meter. Propagation loss was determined using the cutback method. ${ }^{12}$ In order to polish back the waveguides, they were first potted in a transparent wax (Crystalbond). The wax prevented the waveguides from moving and hence from being damaged during polishing. The propagation losses were determined to be $13.4 \pm 0.7 \mathrm{~dB} / \mathrm{cm}$ for TE and $14.6 \pm 0.6 \mathrm{~dB} / \mathrm{cm}$ for TM polarized lights.

It is believed that there are two main reasons for the high propagation losses obtained. These are the sidewall roughness of the waveguide as well as irradiation damage of the waveguide itself during the PBW. It is expected that the irradiation damage could be reduced by modification of the fabrication process to include an annealing step. Oxidizing the waveguides may also help us to alleviate the roughness issue of the waveguide sidewalls.

Additional loss may be a result of the shape and dimensions of the waveguide and the intersection with the support pillars. Therefore, we are currently investigating the fabrication of the square cross section waveguides with smaller dimensions and minimizing interaction with the supports. Future work will also include loss measurements at longer wavelengths. The propagation loss values reported here are rather high; they are nonetheless promising especially when compared to the propagation loss of $\sim 25 \mathrm{~dB} / \mathrm{cm}$ that was measured for early SOI waveguides (e.g., Ref. 13). Therefore, it can be expected that further improvements of the fabrication process will result in much lower propagation loss of the freestanding waveguides, increasing the viability of this technology for mid and far infrared photonic applications.

In this study, we have demonstrated that it is possible to fabricate freestanding waveguides with an air cladding, in a single etch step, using proton beam writing. In the future, the propagation loss measurement of mid infrared wavelengths will be characterized with freestanding waveguides. Our next set of waveguides will be modified to have improved dimensions. Thermal oxidation and annealing processes will also be included in the fabrication in an attempt to reduce the high optical loss presently observed.

${ }^{1}$ http://www.luxtera.com/news_press.htm\#032805.

${ }^{2}$ A. Liu, R. Jones, L. Liao, D. Samara-Rubio, D. Rubin, O. Cohen, R. Nicolaescu, and M. Paniccia, Nature (London) 427, 615 (2004).

${ }^{3}$ H. Rong, R. Jones, A. Liu, O. Cohen, D. Hak, A. Fang, and M. Paniccia, Nature (London) 433, 725 (2005).

${ }^{4}$ V. R. Almeida, C. A. Barrios, R. R. Panepucci, and M. Lipson, Nature (London) 431, 1081 (2004).

${ }^{5}$ Y.-H. Kuo, Y. K. Lee, Y. Ge, S. Ren, J. E. Roth, T. I. Kamins, D. A. B. Miller, and J. S. Harris, Nature (London) 437, 1334 (2005).

${ }^{6}$ R. A. Soref, S. J. Emelett, and W. R. Buchwald, J. Opt. A, Pure Appl. Opt. 8, 840 (2006).

${ }^{7}$ P. Polesello, C. Manfredotti, F. Fizzotti, R. Lu, E. Vittone, G. Lerondel, A. M. Rossi, G. Amato, L. Boarino, S. Galassini, M. Jaksic, and Z. Pastuovic, Nucl. Instrum. Methods Phys. Res. B 158, 173 (1999).

${ }^{8}$ P. Mistry, I. Gomez-Morilla, G. W. Grime, R. Webb, C. Jeynes, R. Gwilliam, A. Cansell, M. Merchant, and K. J. Kirkby, Nucl. Instrum. Methods Phys. Res. B 242, 387 (2006).

${ }^{9}$ M. B. H. Breese, F. J. T. Champeaux, E. J. Teo, A. A. Bettiol, and D. J. Blackwood, Phys. Rev. B 73, 035428 (2006).

${ }^{10}$ E. J. Teo, M. B. H. Breese, E. P. Tavernier, A. A. Bettiol, and F. Watt, Appl. Phys. Lett. 84, 3202 (2004).

${ }^{11} \mathrm{http}: / /$ www.ee.surrey.ac.uk/ibc/index.php?target $=6: 35$.

${ }^{12}$ G. T. Reed and A. P. Knights, Silicon Photonics: An Introduction (Wiley, New York 2004), p. 82, Chap. 4.

${ }^{13}$ B. L. Weiss, G. T. Reed, S. K. Toh, R. A. Soref, and F. Namavar, IEEE Photonics Technol. Lett. 3, 19 (1991). 